\title{
Intraocular Telescopic Implants for Age-related Macular Degeneration Eyes
}

\author{
Isaac Lipshitz
}

Director, Lipshitz Vision Center, Herzlia, Israel

\begin{abstract}
The vast majority of age-related macular degeneration (AMD) eyes (all dry type -90 \% and some of the wet type) do not have any medical treatment that can improve their vision. The only help that they can get are optical solutions and the most adequate are intraocular implanted telescopes. Among the various implanted telescopes that are available (which are reviewed here) the mirror telescope is the newest, smallest, easiest to implant and is the only one that can be used for phakic as well as pseudophakic eyes.
\end{abstract}

\section{Keywords}

Intraocular telescopic implants, AMD, intraocular mirror implant

Disclosure: Isaac Lipshitz is the inventor of the OriLens and the IMT. He own stocks only in the company that developed the OriLems (and not in the company that markets the IMT). No funding was received in the publication of this article.

Open Access: This article is published under the Creative Commons Attribution Noncommercial License, which permits any non-commercial use, distribution, adaptation and reproduction provided the original author(s) and source are given appropriate credit.

Received: 23 November 2015 Published Online: 21 December 2015 Citation: European Ophthalmic Review, 2015;9(2):159-60

Correspondence: Isaac Lipshitz, Director, Lipshitz Vision Center, 46766 Herzlia, Israel. E: lipshitz@netvision.net.il

Optical treatment for improving vision of patients that suffer from agerelated macular degeneration (AMD) is not new, but it is still the only option to improve vision for the majority of AMD patients ( $90 \%$ of AMD are dry-type patients and many of the wet type patients after being treated with injections).

Traditionally, external telescopes and various electronic devices were used in order to help those patients, but they are now seldom used due to the narrow visual field and, more importantly, the relative movement between the eye and the telescope, which causes the patient's visual axis to move in and out of the device. That is why a patient using an external telescope needs to move his/her head in order to screen an image, while the human eye does this by moving the eye, using the extraocular muscles. These head movements are difficult to get used to, especially for the elderly patients.

When implanting an entire telescope inside the eye (in contrast to an external telescope) the implanted telescope moves naturally with the eye movements and there is no relative movement between the eye and the telescope (as there are when using an external telescope). Therefore, the patient's eye scans the reading material or the object normally with no need for head movement for scanningthe image.

Before evaluating the implants for AMD we have first to distinguish between telescopes and magnifiers. Intraocular lens (IOL) magnifiers such as bifocal IOLs with high add power that were recently suggested by Dr Scharioth are not telescopes. They can only add plus power for central vision creating a very short working distance (similar to wearing high power eyeglasses and bringing the reading material nearer), but they are not telescopes and have no effect on distance vision. Whereas telescopic devices create magnification for distance and for near vision and enable normal working distance.

When considering IOL implanted telescopes for AMD we have to distinguish between medical treatment and optical/visual treatment. Other than vitamins, 85-90\% of AMD patients have no medical treatment at all (dry-type AMD). They can be helped only by optical means. Only 10-15\% (wet type) can be assisted by medical treatment and become dry type and qualify for telescopic implantation.

Implantable optical telescopes for AMD are complementary to all other available treatments. They are not intended to replace other treatments, But currently they are the only way to help dry AMD patients improve their visual performance.

This is a new group of patients for any eye surgeon. Surgery is not difficult, but it is not a small incision surgery. But we have to remember that we do not cure the disease or even stop its progress, we just enable the patient to function better with the disease. This treatment requires a long commitment, coordinated with a retinal specialist. The patient selection process is very important and complex.

Currently there are three IOL implantable systems for optical treatment of AMD (see Figure 1):

1. Implantable miniaturised telescope (IMT). This is a miniature Galilean telescope inside a glass tube ( $4.5 \mathrm{~mm}$ in length), which is inserted in the centre of a regular IOL and implanted in the bag and bulging into the anterior chamber through the pupil.

2. The OriLens $^{\circledR}$ (mirror telescopic implant for pseudophakic AMD eyes). The OriLens looks like a regular polymethilemethacrylate 


\section{Figure 1: Schematic Images that Show the Position of the Various Telescopic Systems Inside the Eye}

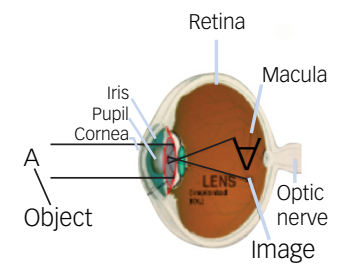

Orilens

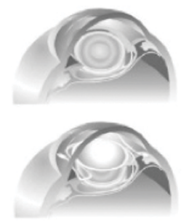

IOL-VIP

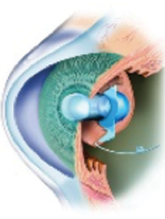

IMT
OriLens = pseudophakic mirror telescopic implant; IMT = implantable miniaturised telescope; IOL-Vip = double (high plus and high minus) intraocular lens implant.

Figure 2: Four Years Follow-up on an Implanted Mirror Telescope in the Posterior Chamber Over an Existing Regular IOL

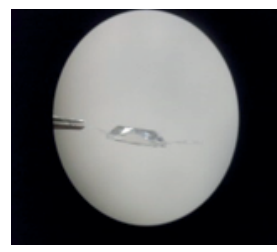

OriLens: Side view

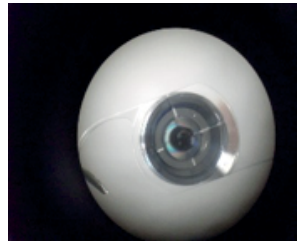

Front view

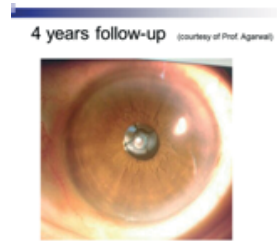

4 years post op
Side view: demonstrates the small central thickness of $1.25 \mathrm{~mm}$ only; Front view: mirror telescope and artificial pupil. IOL = intraocular lens.

(PMMA) IOL having of miniature mirrors embedded inside the IOL. These mirrors are configured, designed and accurately positioned in order to create a precise telescopic effect on the retina. It is implanted in the posterior chamber over an existing IOL. The mirror telescope implant can be used for any type of AMD (wet, dry, scar).

3. The IOL-Vision ImProvement (VIP)\IOL for AMD (IOLAMD) system consists of two IOLs implanted inside an eye, one high minus (over -50.00 D) in the posterior chamber and one high plus (over +50.00 D) in the anterior chamber.

When comparing the three systems the following features should be noted:

The OriLens is the only telescopic implant that can be also used for pseudophakic eyes and the vast majority (over $80 \%$ ) of the patients are already pseudophakic when they require the implant.

The magnification of the OriLens and the IMT is $X 2.5 \mathrm{Mag}$. While the IOL-VIP has only $25 \%$ magnification and this is rarely enough for most AMD eyes.

While the surgical procedure for the IMT is very demanding (a 160-180 opening of the eye and inserting a $4.6 \mathrm{~mm}$ implant into the bag that is bulging into the anterior chamber). The surgical procedure for the OriLens is relatively simple (inserting a piggy back IOL into the sulcus over and existing IOL). There is also a significant difference in the visual field that these telescopes create: the IOL-VIP creates a mild $25 \%$ magnification in the field, while the IMT creates a magnified centre of about $20^{\circ}$ and obscures the peripheral field and the OriLens creates a central magnified image of about $20^{\circ}$, but also preserves some of the peripheral vision (depending on pupil size).

When looking at the endothelial cell count, the OriLens is the easiest to implant and safest as it is entirely implanted in the posterior chamber and does not come in contact with the cornea while the IMT is bulging into the anterior chamber and may compromise the cornea especially during the surgical process (see Figure 2) and the IOL-VIP has a very high plus lens implanted in the anterior chamber coming close to the endothelium.

There are also some differences in the patient selection criteria between the three systems, but for general understanding, here are some of the selection criteria used for the OriLens implantation:

Bilateral AMD or other macular diseases, AMD - dry type, wet type, scar stage or other similar lesions, visual acuity ranges between 20/80 and 20/800 in each eye, no other ocular diseases (other than cataract/pseudophakia, AMD or another macular disease), visual acuity improved for distance and/or near when tested with X2.5 magnification using an external telescope, easy to communicate with, responsible and understands his/her condition, motivated for implantation of the OriLens implant, knows the risks and potential benefits that are involved and is highly motivated to read and improve visual capabilities, ready to sign an informed consent and has successfully undergone all of the pre-op eye examinations.

Some further advantages of the OriLens are: If needed it can be easily explanted, it uses reflective optic elements (mirrors) for creating the magnification thus it is the smallest of them all (only $1.25 \mathrm{~mm}$ central thickness), it can be used for phakic as well as for pseudophakic eyes (by performing a regular cataract procedure and then implanting the OriLens in the same session) and can be used for any type of AMD (dry, wet or scar).

\section{Why should We Consider Adopting the Mirror Telescope into Our Practice?}

The use of implanted telescopes is synergistic to any AMD practice, there is no need for additional equipment or investment, there is a large group of patients with no solutions, retina is the trend today (antivascular endothelial growth factor), the ageing population in western countries increases AMD prevalence, surgery is relatively simple and in some implants also reversible, the results can be predicted preoperatively (by testing the patient with external telescope) and the telescope is additive to any other retinal treatment. 